\title{
ARTICLE \\ In vitro testicular organogenesis from human fetal gonads produces fertilization-competent spermatids
}

Yan Yuan ${ }^{1,2}$, Laihua $\mathrm{Li}^{2}$, Qing Cheng ${ }^{3}$, Feiyang Diao ${ }^{4}$, Qiao Zeng ${ }^{4}$, Xiaoyu Yang ${ }^{4}$, Yibo Wu ${ }^{1}$, Hao Zhang $~^{2}{ }^{2}$, Mingqian Huang $^{2}$ Junqing Chen ${ }^{2}$, Quan Zhou ${ }^{2}$, Yunfei Zhu ${ }^{2}$, Rong Hua ${ }^{2}$, Jianyu Tian ${ }^{2}$, Xin Wang ${ }^{2}$, Zuomin Zhou ${ }^{2}$, Jie Hao ${ }^{5}$, Jinjin Yu', Dong Hua ${ }^{1}$, Jiayin Liu ${ }^{4}$, Xuejiang Guo (D) $^{2}$, Qi Zhou iD $^{5}$ and Jiahao Sha ${ }^{2,3}$

Unlike most organs that mature during the fetal period, the male reproductive system reaches maturity only at puberty with the commencement of spermatogenesis. Robust modelling of human testicular organogenesis in vitro would facilitate research into mechanisms of and factors affecting human spermatogenic failure and male fertility preservation in prepubertal tumor patients. Here, we report successful recapitulation of human testicular organogenesis in vitro from fetal gonadal ridge. Our model displayed the formation of mature seminiferous epithelium and self-renewing spermatogonia. Remarkably, in vitro-derived haploid spermatids have undergone meiotic recombination, and showed increased genetic diversity as indicated by genetic analysis. Moreover, these spermatids were able to fertilize oocytes and support subsequent blastocyst formation. The in vitro testicular organogenesis system described here will play an important role in elucidating the regulation of human testis development and maintaining male fertility in prepubertal cancer patients.

Cell Research (2020) 30:244-255; https://doi.org/10.1038/s41422-020-0283-z

\section{INTRODUCTION}

The mammalian testis produces sperm by spermatogenesis and secretes hormones. Testicular organogenesis is a complex process involving morphologic and functional phases in different testicular compartments that culminate in testis maturation at puberty. ${ }^{1-3}$ Proliferation and differentiation of germ cells occur in close contact with somatic cells. Real-time dynamic studies of the maturation of somatic and germ cells and their interactions during organogenesis could therefore provide valuable insight into human reproductive biology.

The ability to continuously produce sperm is essential for male fertility. ${ }^{4}$ Approximately $8 \%-12 \%$ of couples worldwide are infertile, and male infertility contributes to more than half of all cases with spermatogenesis failure representing a leading causal factor. ${ }^{5}$ Spermatogenesis is susceptible to the effects of genetic mutations, endocrine disorders, and environmental endocrine disruptor chemicals, among others, but there is little understanding of the mechanisms of its pathogenesis. Moreover, the incidence of childhood cancer ranges from 1.0 to 2.5 per 1000 children. ${ }^{6}$ And the risk of childhood cancer could be significantly increased by the use of a recently widely used assisted reproductive technology, frozen embryo transfer, according to Denmark data. ${ }^{7}$ Approximately $30 \%$ of patients receiving gonadotoxic cancer treatments are permanently infertile. ${ }^{8}$ As a result, one in about 5,000 young men is at risk of infertility due to cancer therapy. ${ }^{9}$ In addition to the unpredictable impact on germ cells, ${ }^{10,11}$ chemoradiotherapy causes numerous gene mutations in somatic cells. ${ }^{12-14}$ One option for fertility preservation in male cancer patients is testicular tissue cryopreservation. ${ }^{15-18}$ Consequently, various approaches that restore spermatogenesis from such tissue are being explored, including cell transplantation, autotransplantation of testicular tissue, or in vitro spermatogonial maturation, but current systems have to date been unable to produce sperm by meiosis. An in vitro system for testicular organogenesis would enable investigation of genetic, epigenetic, and environmental factors that shape germ cell and testicular somatic cell development. It would also provide insight into the pathological mechanisms of various spermatogenesis disorders. Additionally, such an in vitro system could provide technical means of preserving fertility in prepubertal cancer patients undergoing tumor chemotherapy and radiotherapy, as sperm could be generated from preserved tissue biopsies without the need for transplantation.

Successful recapitulation of testicular organogenesis in vitro requires the completion of meiosis and the production of functional haploid gametes. To prevent misconceptions of meiosis in vitro, reproductive biologists have therefore defined a panel of "gold standard" criteria that must be met by in vitroderived gametes to show that meiosis has occurred in vitro. ${ }^{19}$ These criteria reflect key events of meiosis and include correct nuclear DNA content, normal chromosome content and organization, recombination, and the ability to produce viable

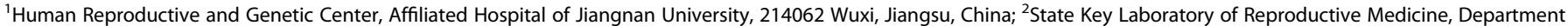

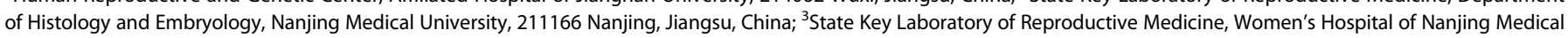

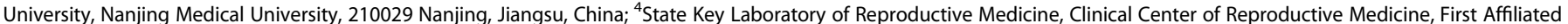

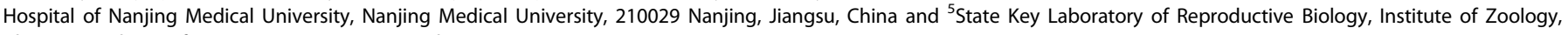
Chinese Academy of Sciences, 100101 Beijing, China

Correspondence: Jiahao Sha (shajh@njmu.edu.cn) or Qi Zhou (zhouqi@ioz.ac.cn) or Xuejiang Guo (guo_xuejiang@njmu.edu.cn)

These authors contributed equally: Yan Yuan, Laihua Li, Qing Cheng, Feiyang Diao, Qiao Zeng

Received: 11 July 2019 Accepted: 3 February 2020

Published online: 21 February 2020 
euploid offspring. Despite multiple attempts, successful in vitro meiosis conforming to these "gold standard" criteria has not been achieved in human.

Here, we report the successful recapitulation of human testicular organogenesis in vitro from cultured fetal gonads. Upon exposure to morphogens and sex hormones, human germ cells recapitulate spermatogenesis together with meiosis in vitro, demonstrating imprint erasure, synapsis and recombination hallmarks, correct chromosome content and structures, and maturation of somatic cells. The successful testicular organogenesis in culture is expected to provide in vitro models for further studies of human reproductive biology and male fertility preservation.

\section{RESULTS}

Establishing a robust culture system for human testicular organogenesis in vitro

Human germ cells arrive at genital ridge at week 5 , and sexual differentiation completes between week 6 and week $9 .^{20}$ Previous studies identified three phases of male germ cell development: migrating phase (before the 4th week), gonadal mitotic phase (4 to 25 weeks), and mitotic arrest phase (9 to 25 weeks). ${ }^{21}$ Before in vitro cultures were established, we first characterized the properties of germ cells in gonads harvested from aborted human male fetuses (12 to 19 weeks), which contained mitotic active and mitotic arrest germ cells (Supplementary Information, Fig. S1a-c). Immunostaining revealed that DDX4-positive male germ cells had large nuclei with lower intensity of Hoechst staining than surrounding cells. Twelve-week gonads contained DDX4-positive germ cells, approximately half of which co-expressed OCT4, BLIMP1, AP2 $\gamma$, key transcription factors for primordial germ cells (PGCs), and Ki-67, an antigen for proliferating cells (Supplementary Information, Fig. S1b). PLZF was expressed in some of the 12-week germ cells (Supplementary Information, Fig. S1b). The marker PLZF is expressed by (pro)spermatogonial cells and regulates selfrenewal and maintenance of the stem cell pool. ${ }^{22,23}$ Nineteenweek gonads displayed an increased number of PLZF-expressing germ cells, while fewer germ cells co-expressed BLIMP1, OCT4 and AP2 $\gamma$ relative to week 12 . Furthermore, most 19-week DDX4positive germ cells lacked $\mathrm{Ki}-67$ expression (Supplementary Information, Fig. S1b). Thus, from 12 to 19 weeks, male PGCs gradually achieve mitotic arrest differentiating into advanced germ cells, which is either preceded by or coincident with the upregulation of PLZF expression and the repression of key PGC markers. The above results indicate that germ cells of week 12 to 19 are in the stage of transition from early PGC to late PGC further changing to prospermatogonia.

Using a standard gas-liquid interphase method, ${ }^{24}$ we cultured gonad tissue fragments of approximately $3 \mathrm{~mm}$ in diameter on small agarose gels semi-submerged in medium (Fig. 1a, b). To reproduce the temperature of the scrotum, which is approximately 3 degrees below core temperature, cultures were maintained at $34^{\circ} \mathrm{C}$. The culture medium was modified from a method developed by our laboratory ${ }^{25}$ and contained glial cell line-derived neurotropic factor (GDNF), basic fibroblast growth factor (bFGF), and epidermal growth factor (EGF), which were essential for the proliferation of germ cells. Co-immunostaining for DDX4 and GATA4 showed that compared with tissues cultured in the medium with all cytokines and hormones (complete testis culture medium), tissues cultured in the medium without cytokines or hormones (testis culture medium) or complete testis culture medium with additional retinoic acid (RA) (complete testis culture medium + RA) contained lower levels of DDX4-positive germ cells at day 30 (Supplementary Information, Fig. S3a, b). Tissues cultured in the presence of RA did not contain detectable levels of DDX4-positive germ cells at day 30 of culture and were apoptotic as shown by TUNEL staining (Supplementary Information, Fig. S3c).
To characterize human testis organogenesis during different stages of in vitro culture, we performed immunostaining with antibodies for the following cell type-specific markers: DDX4 for germ cells, PLZF for (pro)spermatogonia, ${ }^{23}$ SYCP3 for spermatocytes, PRM1 for spermatids, SOX9 for Sertoli cells, and CYP17A1 for Leydig cells (Fig. 1c). With uncultured tissue (day 0, 12-week genital ridge) for comparison, immunostaining showed that, between day 10 and 50, cultured tissues developed seminiferous tubules filled with DDX4-positive germ cells arranged from the basal membrane to central lumen (Fig. 1c). The proportion of DDX4-positive cells did not significantly fluctuate with incubation time (Supplementary Information, Fig. S1d). The proportion of PLZF-positive cells increased significantly at day 10 and subsequently remained at similar levels at day 30 and 50 of culture. Immunostaining of human testis with DDX4 and PLZF was used as a positive control (Supplementary Information, Fig. S2a). Expression of SYCP3 was observed in the testicular tissue from day 10 to 50 , suggesting that germ cells have entered the process of meiosis (Fig. 1c; Supplementary Information, Fig. S1d). Furthermore, staining for PRM1 confirmed the appearance of spermatids at day 30 and day 50 of culture (Fig. 1c). Staining for PNA also supported the appearance of spermatids in the cultured tissue (Supplementary Information, Fig. S5c). SYCP3-positive cells were detected at day 10 of culture, PRM1-positive cells were detected at day 30 of culture, and their proportions remained at these levels for the subsequent culture time (Supplementary Information, Fig. S1d). The above immunofluorescence (IF) results showed gradual establishment of seminiferous epithelium, with both spermatogonia and spermatocytes after ten days of culture, and the presence of spermatogonia, spermatocytes and spermatids after 30 and 50 days of culture. And the hematoxylin and eosin (H\&E) staining also showed the presence of spermatogonia, spermatocytes and spermatids in the cultured tissue at day 50 (Fig. 1d). Staining for the markers, SOX9 and CYP17A1, delineated the presence of Sertoli cells and Leydig cells in the testicular tissue from day 0 to 50 (Fig. 1C). The presence of spermatogonia, spermatocytes, spermatids, Sertoli cells, and Leydig cells in the same tubule indicated the establishment of spermatogenic epithelium and the completion of organogenesis. Of the 45 used gonad samples, 44 were subjected to culture studies, 23 successfully developed spermatid-like cells after culture as determined by morphological analysis or flow cytometry. Of the above 23 tissues, 14 were shown to develop haploid cells by flow cytometry, and sorted haploid cells from 11 tissues were further subjected to short tandem repeat (STR), fluorescent in situ hybridization (FISH), IF or copy number variation (CNV) analysis. Nine rest tissues were found to develop Prm1- or PNA-positive cells by IF analysis. Due to the variances of sample quality, 21 samples gradually underwent apoptosis and could not produce spermatids. In summary, we describe a culture technique that supports testicular organogenesis, including the development of embryonic testis tissue, meiosis of spermatocytes and production of spermatids in vitro.

\section{Sertoli and Leydig cells demonstrate in vitro maturation}

Sertoli cell proliferation and anti-Müllerian hormone (AMH) expression are regulated by follicle-stimulating hormone (FSH). ${ }^{2}$ Sertoli cells in fetal gonads show typical immature oval and elongated nuclei with a regular shape. ${ }^{26}$ To promote maturation of Sertoli cells, we used FSH as previously reported by our laboratory. ${ }^{25}$ Following culture for 30 days, Sertoli cells exhibited intense AR staining (Fig. 2a), suggesting differentiation of Sertoli cells similar to human testis control (Supplementary Information, Fig. S4b). In the embryonic genital ridge cultures, AMH expression was high at baseline but nearly absent after 30 days of culture (Fig. 2b). Furthermore, many of the GATA4-positive Sertoli cells exhibited reduced proliferation as represented by decreased Ki-67 signal co-localization (Supplementary Information, Fig. S4a), 
a

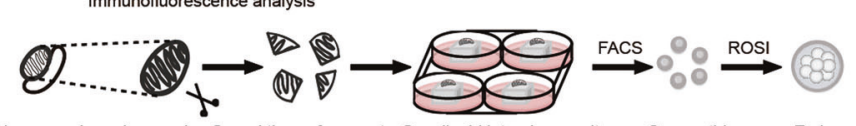

Human embryonic gonad Gonad tissue fragments Gas-liquid interphase culture Spermatids

Embryo b

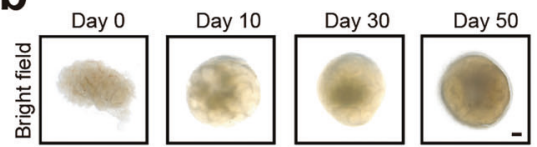

C
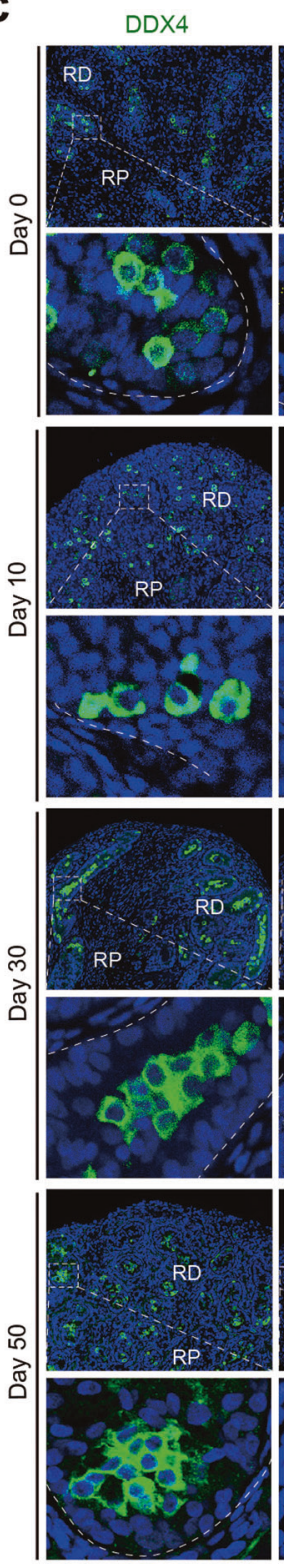

PL 7F
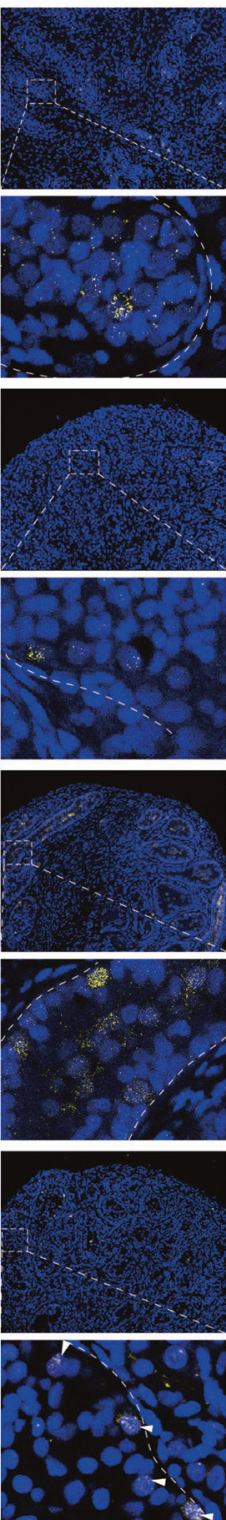

SYCP3
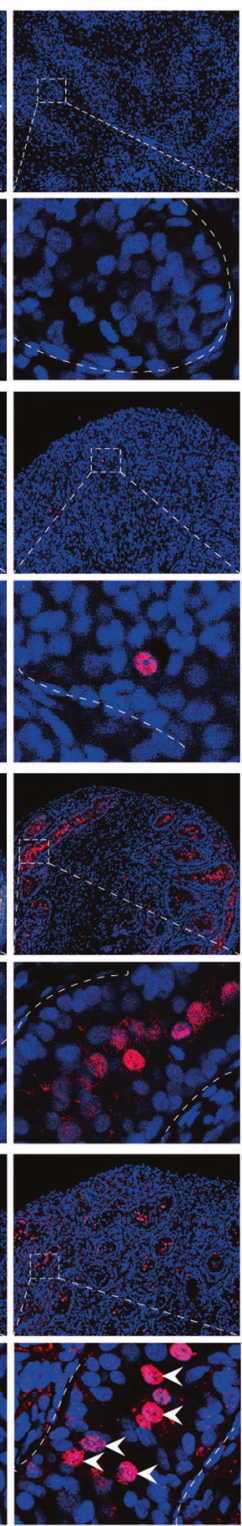

PRM1
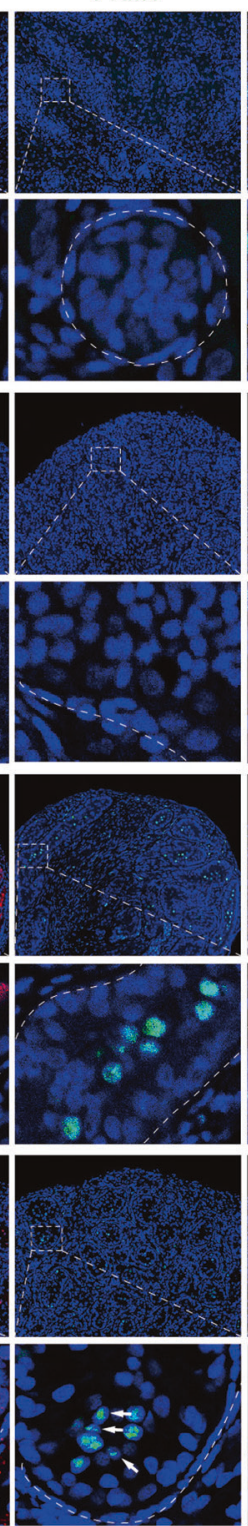

CYP17A1
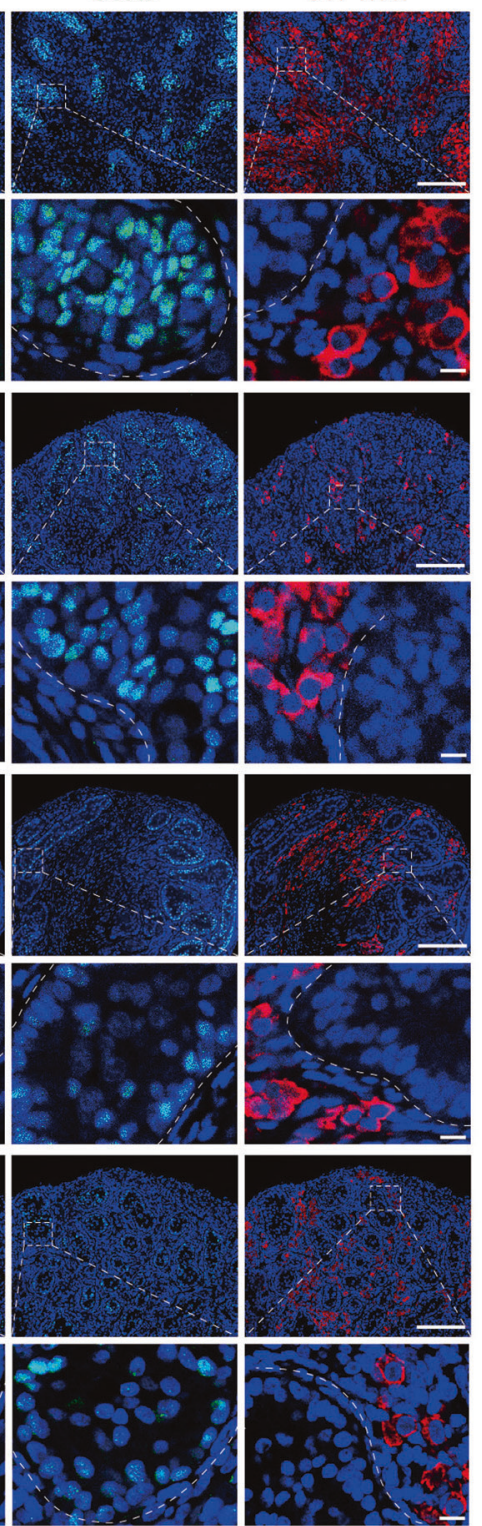

d

Spermatocyte

Spermatid

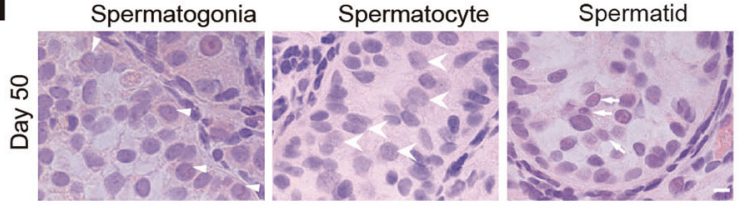

Fig. 1 In vitro organogenesis of male human fetus gonads. a Schematic representation of the technique used for human testicular organogenesis from embryonic gonads. b Bright-field images of 0-, 10-, 30-, and 50-day cultures. Scale bar = $1 \mathrm{~mm}$. c Immunostaining of 0-, 10-, 30-, 50-day cultured gonadal tissues for DDX4 (green), PLZF (yellow) (triangle), SYCP3 (red) (arrowhead), PRM1 (green) (arrow), SOX9 (green), and CYP17A1 (red). Scale bars of low magnification $=100 \mu \mathrm{m}$. Scale bars of partial magnification $=10 \mu \mathrm{m}$. DNA was counterstained with Hoechst 33342 (blue). RP, Region proximal to mesonephros; RD, Region distal to mesonephros. d Consecutive H\&E staining of the same section of 50-day cultured tissue used in IF analysis (c). Spermatogonia (triangle), spermatocytes (arrowhead), and spermatid-like cell (arrow) are indicated; Scale bars, $10 \mu \mathrm{m}$. 
a

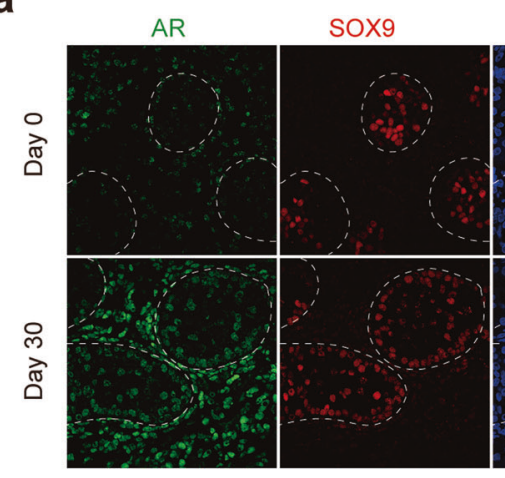

C

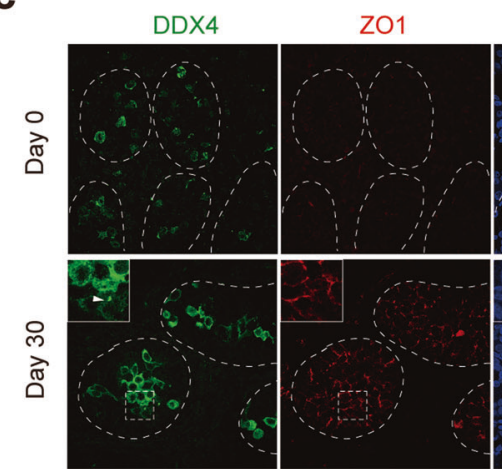

e
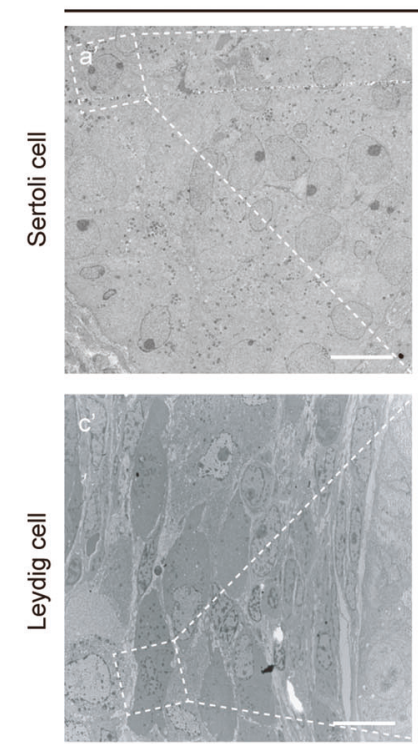

Day 0
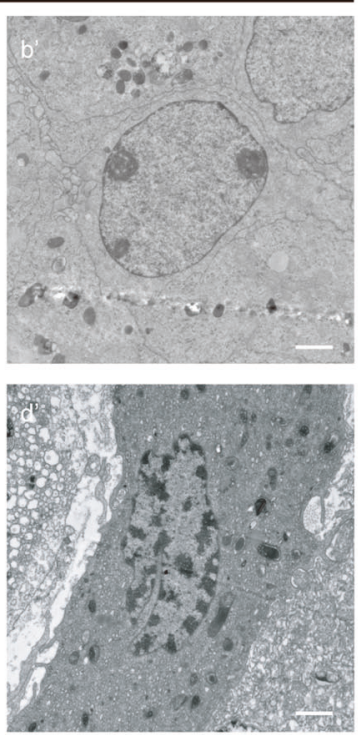

b
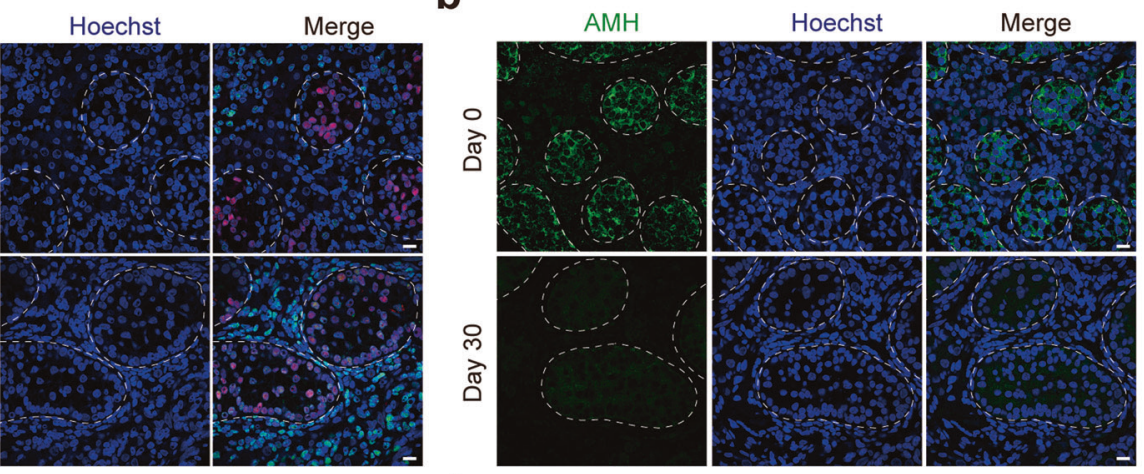

d
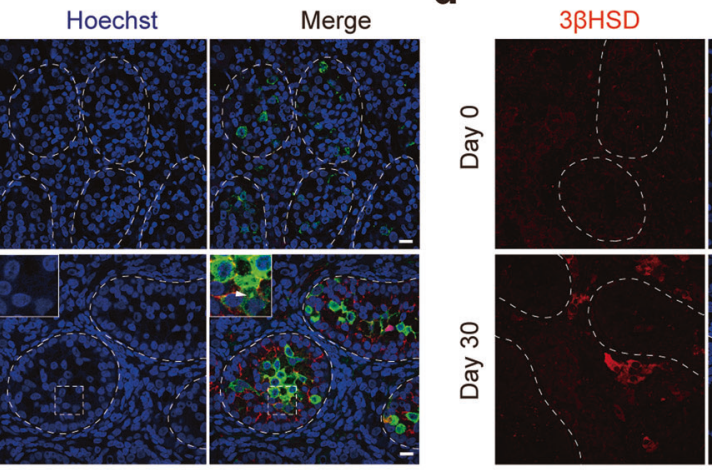

Hoechst

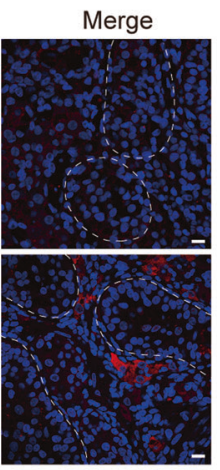

f

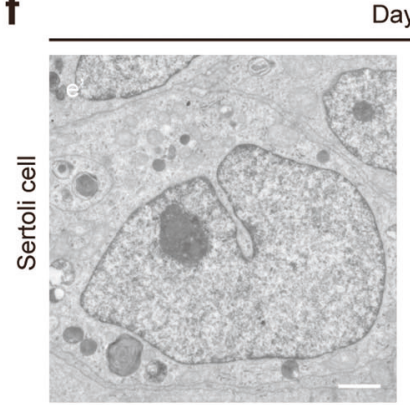

Day 30

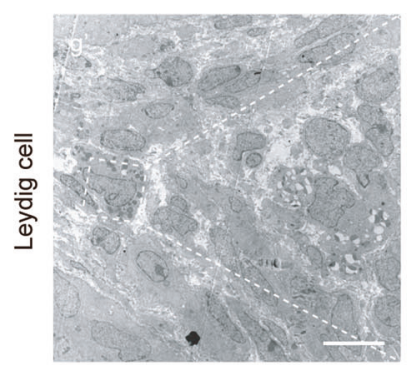

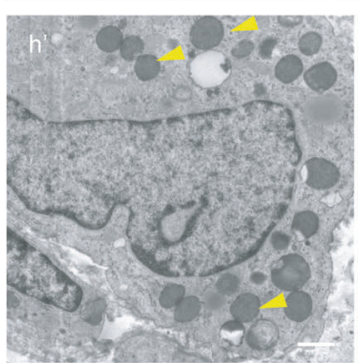

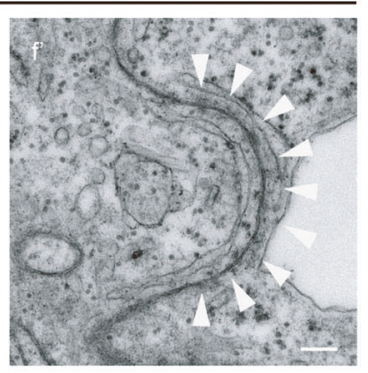

Fig. 2 Sertoli and Leydig cell maturation during human testis organogenesis in vitro. a Immunostaining of 0- and 30-day cultured gonadal tissues for AR (green) and SOX9 (red). Scale bars $=10 \mu \mathrm{m}$. DNA was counterstained with Hoechst 33342 (blue). b Detection of Sertoli cells in 0and 30-day cultured tissues by immunostaining for $\mathrm{AMH}$ (green). Scale bars $=10 \mu \mathrm{m}$. DNA was counterstained with Hoechst 33342 (blue). c Immunostaining of 0 - and 30-day cultured tissues for DDX4 (green) and ZO1 (red). Scale bars = $10 \mu \mathrm{m}$. DNA was counterstained with Hoechst 33342 (blue). Chromatoid body (white triangle). d Immunostaining of Leydig cells for $3 \beta$-HSD (red) after 0 and 30 days in culture. Scale bars $=10 \mu \mathrm{m}$. DNA was counterstained with Hoechst 33342 (blue). e, $\mathbf{f}$ Transmission electron microscopy shows ultrastructural features of Sertoli and Leydig cells from day 0 and 30 cultured gonadal tissues. Tight junction between Sertoli cells (white triangles) and lipid droplets in the Leydig cells (yellow triangles) are indicated. Scale bars $=10 \mu \mathrm{m}\left(a^{\prime}, c^{\prime}, g^{\prime}\right) ; 1 \mu \mathrm{m}\left(b^{\prime}, d^{\prime}, e^{\prime}, h^{\prime}\right) ; 200 \mathrm{~nm}\left(f^{\prime}\right)$.

suggesting Sertoli cell maturation. Staining for DDX4 and ZO1 of cultured tissues and human testis control indicated the establishment of the blood-testis barrier (BTB) (Fig. 2c; Supplementary Information, Fig. S4c) that divided the seminiferous epithelium into the basal and adluminal compartments, creating unique microenvironments supporting self-renewal and differentiation of spermatogonia.
Transmission electron microscopy (TEM) revealed ultrastructural features consistent with immature Sertoli cells in baseline gonads (primary gonads that have not been cultured), with ovoid nuclei and small nucleoli (Fig. 2e), while 30-day cultures demonstrated features consistent with mature Sertoli cells that possessed clear cytoplasm, irregularly-shaped nuclei, tight junctions, and abundant vesicles near the endoplasmic reticulum (Fig. 2f; 
Supplementary Information, Figs. S2b, S4e). Consistent with protein expression, AMH transcripts decreased, and AR transcripts increased during culture (Supplementary Information, Fig. S4d). Thus, decreased $\mathrm{AMH}$ expression during the in vitro time course coincides with decreased mitosis and increased AR expression, suggesting maturation of the spermatogenesis-supporting Sertoli cell.

Next, we profiled evidence of androgen secretion, as androgen secreted by Leydig cells is necessary for spermatogenesis. ${ }^{27}$ Immature Leydig cells in baseline fetal gonads showed no obvious expression of $3 \beta$-hydroxysteroid dehydrogenase (3 $\beta$-HSD), which is a marker of steroidogenic activity, whereas Leydig cells after 30 days of culture displayed strong cytoplasmic $3 \beta-H S D$ expression (Fig. 2d). This finding was confirmed by elevated $3 \beta-H S D$ transcript levels (Supplementary Information, Fig. S4d). CYP17A1, another enzyme required for androgen synthesis, was expressed by Leydig cells at baseline and persisted during culture (Fig. 1c). TEM revealed the presence of numerous cytoplasmic lipid droplets, a feature consistent with functional maturation of the Leydig cells, after 30 days of culture (Fig. 2e, f; Supplementary Information, S4e). These molecular and morphologic data suggest that Leydig cells in cultured tissues acquire the ability to secrete androgen in vitro.

In vitro establishment of spermatogenesis

Spermatogenesis in adult testis includes mitosis of spermatogonia, meiosis of spermatocytes, and spermiogenesis of spermatids to form functional sperm. The self-renewal of spermatogonia ensures sustained spermatogenesis. Immunostaining of $0-, 10-, 30-$ and $50-$ day cultures confirmed the presence of PLZF and Ki-67 doublepositive undifferentiated spermatogonia in the seminiferous tubules, which indicated that PLZF-positive spermatogonia could self-renew in vitro (Fig. 3a, b). Immunostaining also showed Lin28a-positive spermatogonia in the seminiferous tubules (Supplementary Information, Fig. S5a). We next examined progression of meiosis in the cultured tissues and adult human testis control by immunostaining for $\mathrm{YH} 2 \mathrm{AX}$, SYCP3 and SYCP1 (Fig. 3c; Supplementary Information, S2a). These marker proteins exhibited distinct expression and co-localization patterns during chromosomal synapsis in spermatocytes. Specifically, the distribution of $\gamma \mathrm{H} 2 \mathrm{AX}$ and SYCP3 recapitulated meiotic progression in vivo. $^{28}$ In vitro cultured tissues contained spermatocytes positive for both $\mathrm{YH} 2 \mathrm{AX}$ and SYCP3 (Fig. 3c), with XY body stained by $\gamma \mathrm{H} 2 \mathrm{AX}$ (Supplementary Information, Fig. S5b). Furthermore, meiotic recombination of homologous chromosomes was evidenced by co-localization of SYCP3 and MLH1, with spot-like MLH1 signals appearing in the nuclei of SYCP3-positive spermatocytes (Fig. 3c). Human adult testis was used as a positive control (Supplementary Information, Fig. S2a). However, these immunofluorescence data are still not sufficient to support that the germ cells were undergoing meiosis in vitro.

TEM examination revealed the formation of seminiferous epithelium, presence of spermatogonia beneath the basement membrane of the seminiferous tubules, similar to those observed in human adult testis, and primary spermatocytes containing synaptonemal complexes (Fig. 3d; Supplementary Information, Figs. S2b, S5g). Additionally, acrosome vesicles and early tail structures could also be observed in the seminiferous tubules (Fig. 3d; Supplementary Information, Fig. S5g). H\&E staining also showed the presence of spermatocytes in the seminiferous tubules after 10-day culture, and spermatid-like cells after 50-day culture, which were similar to the in vivo spermatids (Supplementary Information, Fig. S5h).

The above findings were also supported by flow cytometric analysis. Baseline gonadal tissues contained $2 \mathrm{C}$ or 4 C DNA content. Flow cytometric results from the beginning of in vitro culture to a maximum of 134 days were collected to indicate development of haploid cells during testicular organogenesis (Supplementary
Information, Table S1). After 10 days of culture, the proportion of 4C cells increased (Fig. 3e) in accordance with the appearance of spermatocytes in IF analysis (Fig. 1C). Haploid (1 C) cells were first detectable in 30-day cultures and represented about $10 \%$ of the total fraction of cells in cultures beyond 50 days (Fig. 3e, f), indicating continuous spermatogenesis. FACS analysis showed that after approximately 50 days of culture, tissues contained an average proportion of $7.24 \%$ haploid cells ( 11 biological replicates; Supplementary Information, Table S2).

Induced spermatids exhibit key genetic and epigenetic features Next, we characterized purified haploid cells by FACS, IF and FISH. ACROSIN, a sperm-specific protein localized to the acrosomal matrix surrounding the spermatid nucleus, was expressed by $12.6 \%$ of FACS-purified haploid cells (Fig. 4a). FACS-purified haploid cells were also positive for PNA, another marker of acrosome (Supplementary Information, Fig. S5d). Furthermore, IF analysis of cultured tissues showed spermatids with DDX4-positive chromatoid body (Fig. 2c). Nuclei of 13 FACS-purified haploid cells from 55-day cultures were profiled by FISH analysis. Of the 13 nuclei analyzed with the FISH probe set $1(13,21), 10$ cells $(76.9 \%)$ displayed a positive signal in chromosome 13 and a positive signal in chromosome 21, which confirmed a haploid state. Of 11 nuclei analyzed by probe set $2(x, y), 9$ cells displayed a positive signal for chromosome $\mathrm{X}$, and 1 cell displayed a positive signal for chromosome $\mathrm{Y}$, which confirmed a haploid state in 10 cells (90.9\%) (Fig. 4b). Furthermore, FISH analysis using sex-specific chromosomal probes confirmed the haploid state of spermatids sorted by FACS after 134-day culture (Supplementary Information, Fig. S5e, f).

Besides the above cytological analysis of meiotic recombination, we also performed the genetic analysis. We used single-cell whole genome sequencing to profile CNV and assess genomic integrity in FACS-purified spermatids derived from our culture system and diploid control cells from aborted fetal skin cell. Of 10 spermatids, 9 cells displayed a haploid genome without structural abnormalities, and one cell displayed a chromosome 12 duplication event (Fig. 4c).

Chromosome recombination results in increased genetic diversity after meiosis, which can be tested by genetic analysis taking advantage of human genetic variations. However, it has not been used in the studies of in vitro meiosis in inbred model organisms. ${ }^{24,25}$ As the "Gold Standards" explained, cytological or molecular detection of the expression of meiotic proteins is not sufficient to prove that putative germ cells are executing meiosis. ${ }^{19}$ Analysis of increased genetic diversity caused by homologous recombination could be a better evidence for completion of meiotic homologous recombination, and better support the "Gold Standards". ${ }^{19}$ We compared human STR profiles of in vitro-derived spermatids to corresponding fetal somatic cells using the Applied Biosystems Identifier Kit. For analysis, we selected the autosomal chromosomes 1 and 6 based on the presence of multiple STR sites which would appear in different combinations due to recombination events (Fig. 4d). The somatic cell sample contained alleles 15 , 16 in D1S1677 locus and 10, 13 in D1S1627 on chromosome 1, and alleles 14, 15 in D6S474 and 10, 12 in D6S1017 on the chromosome 6 . In the corresponding in vitro-derived spermatids, we observed the combinations of alleles 15 and 10, and 16 and 10 on the chromosome 1, indicating recombination between D1S1677 and D1S1627. We also found recombination between D6S474 and D6S1017, by detecting combinations of alleles 15 and 10, and 14 and 10 on chromosome 6 (Fig. 4d). Thus, in vitro-derived spermatids displayed evidence of chromosomal recombination events resulting from meiosis.

Imprinted genes are characterized by epigenetic modifications that differentiate maternal and paternal alleles. These modifications are established during gametogenesis and include DNA methylation of imprinting control elements. Bisulfite sequencing 
a

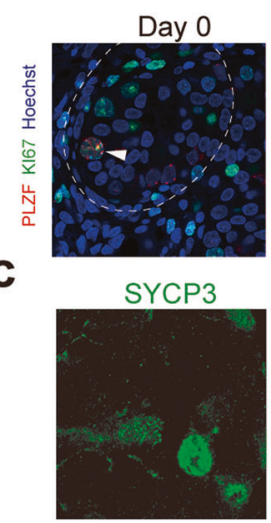

SYCP3

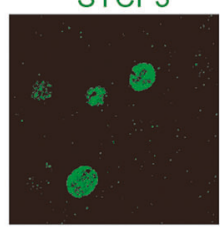

SYCP1

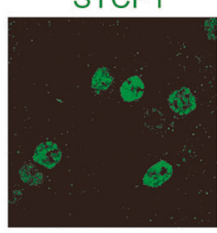

d

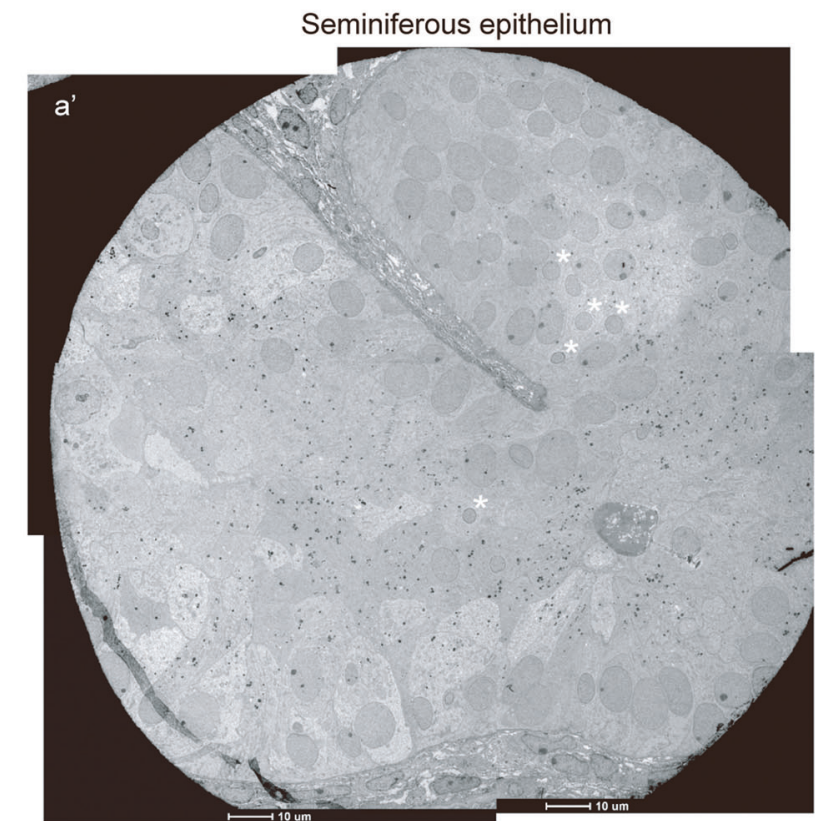

Day 10

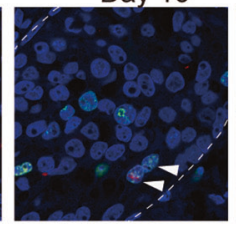

$y+2 A X$

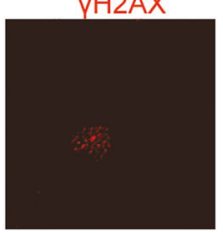

MLH1

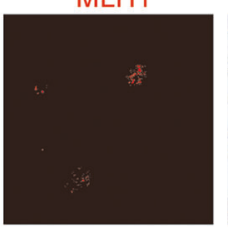

Hoechst
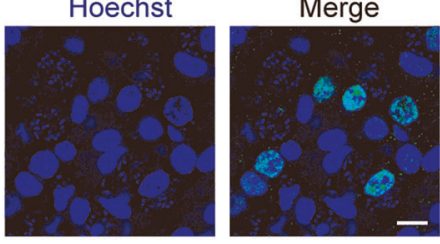

Hoechst
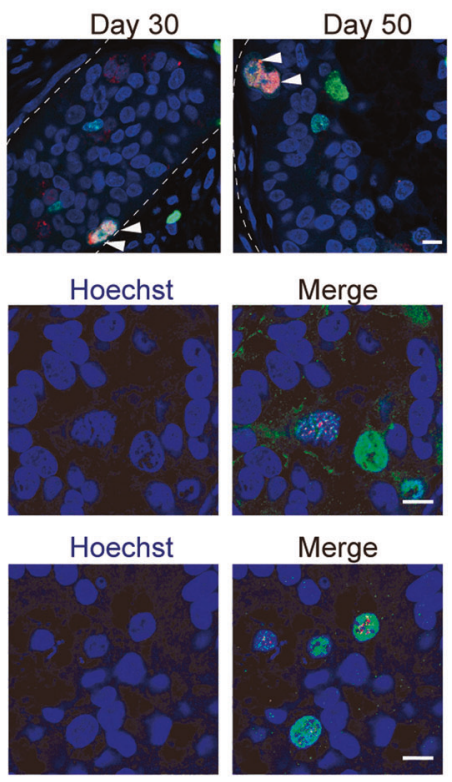

Hoechst
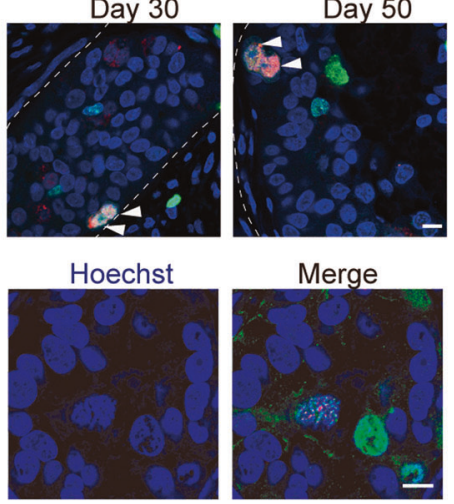

Merge b

e

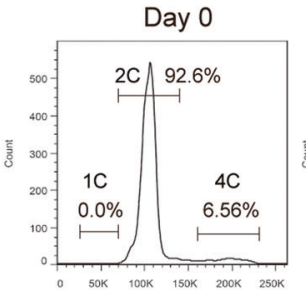

Day 30

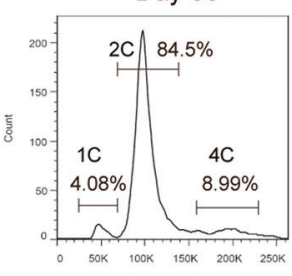

Hoechst

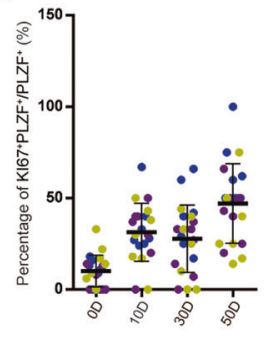

f

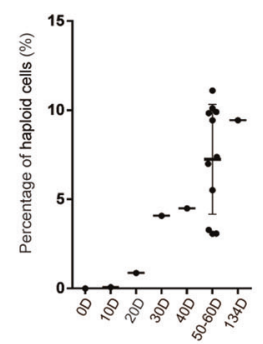

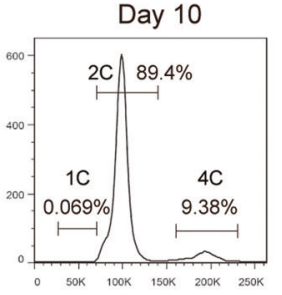

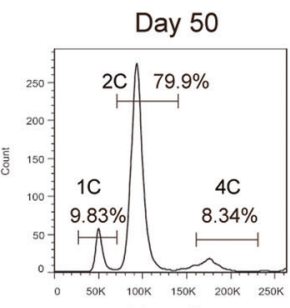

Hoechst

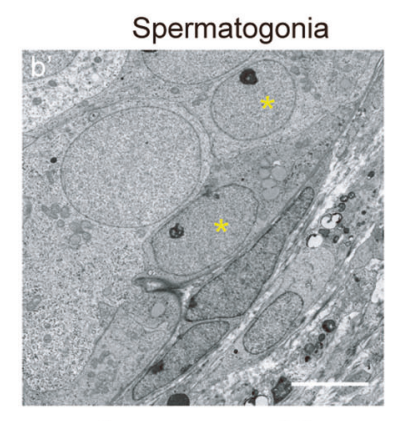

Acrosome vesicle

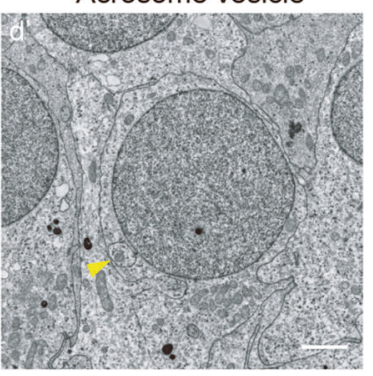

Spermatocyte

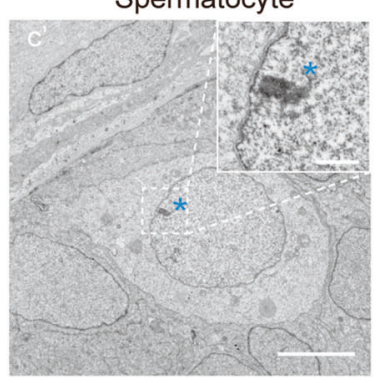

Tail structure

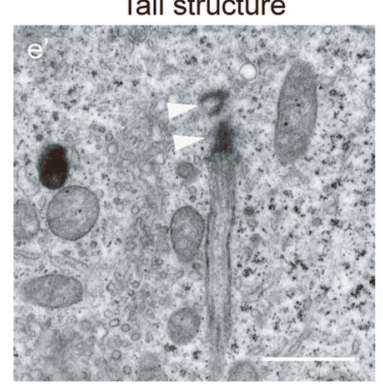

Fig. 3 Successful spermatogenesis of germ cells during human testis organogenesis. a Immunofluorescent labeling of 0-, 10-, 30- and 50day cultured gonadal tissues co-labeled for PLZF (red) and Ki67 (green). PLZF ${ }^{+}$Ki67 ${ }^{+}$cells (white triangles). Scale bar $=10 \mu \mathrm{m}$. $\mathbf{b}$ Percentage of PLZF-positive KI67-positive cells from three biological replicates. (Each dot represents the percentage in the cross section of a seminiferous tubule, different colors of the dots represent different specimens). c Immunostaining of cultured tissues for SYCP3 (green), $\gamma \mathrm{H} 2 \mathrm{AX}$ (red), MLH1 (red) and SYCP1 (green). DNA was counterstained with Hoechst 33342 (blue). Scale bar = 10 um. d Transmission electron microscopy shows ultrastructural findings of seminiferous epithelium. Spermatids: white stars; spermatogonia: yellow stars; primary spermatocytes with nuclear synaptonemal complexes: blue stars; spermatids with acrosome vesicles: yellow triangle; and early tail structure: white triangles. Scale bars, $10 \mu \mathrm{m}(\mathbf{a}) ; 2 \mu \mathrm{m}$ (b, d); $200 \mathrm{~nm}$ (c); $1 \mu \mathrm{m}$ (e). e DNA content distribution of cells from 0- to 50-day cultured tissues by FACS. Haploid (1C), diploid (2C), and tetraploid (4C) cells are indicated. $\mathbf{f}$ Percentages of haploid cells in FACS analysis of 11 cultured samples. 
a

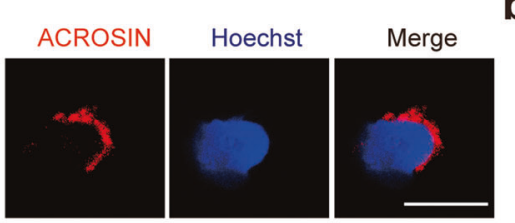

b

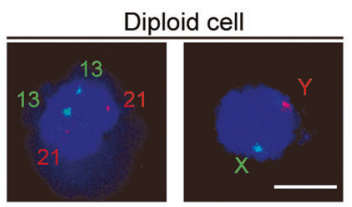

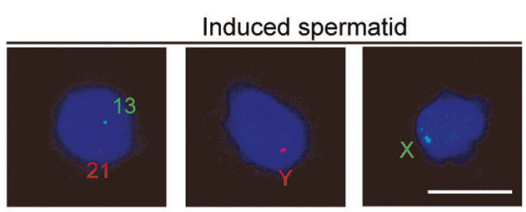

C

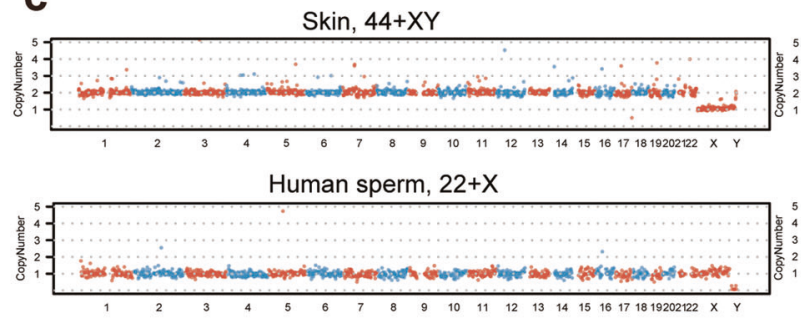

Skin, $44+X Y$

d
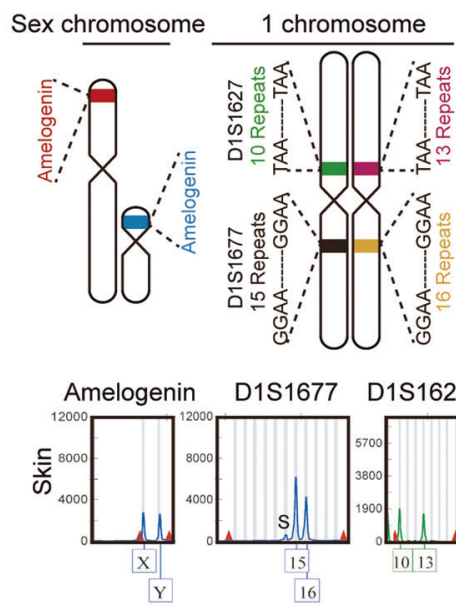

D1S1677

D1S1627

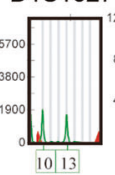

16
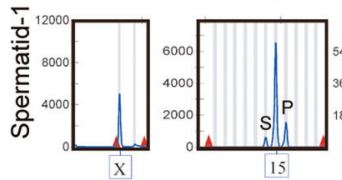

15
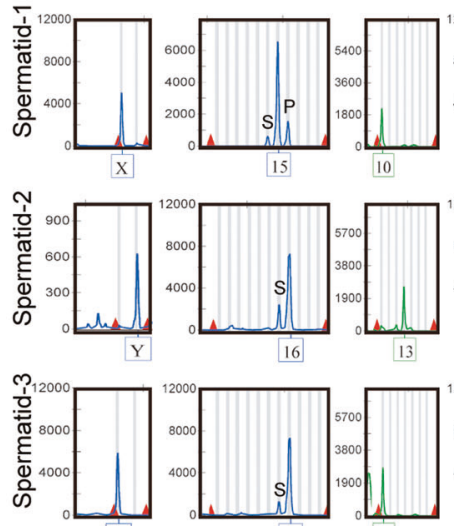

$\mathrm{x}$
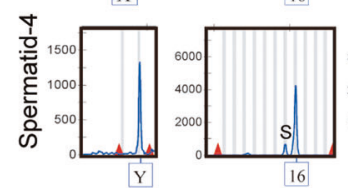
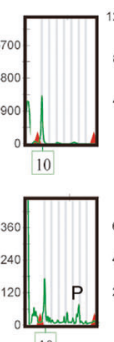

6 chromosome

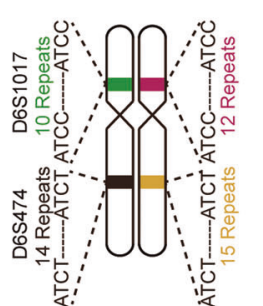

D6S474

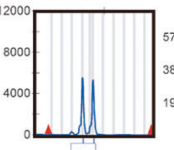

14]

15
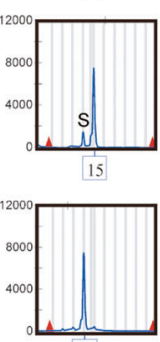

14

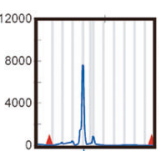

14

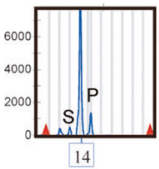

D6S1017
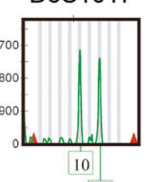

$\frac{10}{12}$

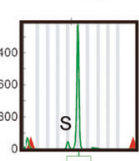

$\sqrt{10}$
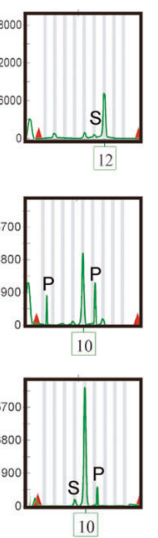

Induced spermatid, $22+X$

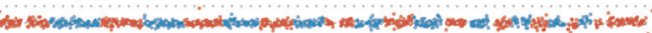

Induced spermatid, $22+X$

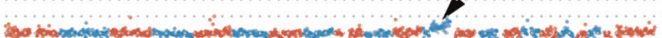

Fig. 4 Key genetic and epigenetic properties of induced spermatids. a Immunostaining for ACROSIN in haploid cells isolated from cultured tissues by FACS. Scale bars $=10 \mu \mathrm{m}$. b FISH analysis using somatic probes specific to chromosomes 13 and 21 , or sex probes specific to chromosomes $X$ and $Y$ for diploid and induced spermatids. Scale bars $=10 \mu \mathrm{m}$. c Genomic DNA sequencing of diploid cells from aborted fetal skin cell, in vivo spermatid and in vitro-derived single spermatid sorted by FACS. Abnormal copy number changes in chromosome 12: black arrow. d Alleles of STR loci in aborted fetal somatic skin cells and induced spermatids depict chromosome separation and homologous recombination (left). Schematic map of chromosomal STR loci and separation of chromosomes after meiosis (right). S: Stutter peak; P: Pull down peak. e Bisulfite sequencing of differentially methylated regions (DMRs) of H19 and SNRPN in human round spermatids, and induced spermatids. White and black circles indicate unmethylated and methylated CpGs, respectively. 
revealed male germ cell-specific differential methylation at the paternally imprinted $\mathrm{H} 19$ and maternally imprinted SNRPN loci, comparable with that of in vivo-derived human sperms (Fig. 4e; Supplementary Information, Table S3). These data demonstrate that in vitro-derived spermatids established the normal methylation status of imprinted genes, that is, hypermethylation of the paternal imprint and hypomethylation of the maternal imprint.

In vitro-derived spermatids fertilize oocytes and support early embryo development

The function of in vitro-derived spermatids was evaluated by round spermatid injection (ROSI) to examine the ability to support fertilization and early embryonic development. Spermatids derived from fetal gonadal tissues originating from week 12 and cultured for 55 days were selected for ROSI. Immature oocytes, including metaphase I or germinal vesicle (GV) oocytes, were subjected to in vitro maturation (IVM). Sixteen metaphase II oocytes with polar body formation after IVM were injected with in vitro-derived spermatids. Two embryos developed to the 2-cell stage, and subsequently formed one 8-cell-stage embryo and one blastocyst (Fig. 5a and Table 1). As a control, we performed ROSI of 9 IVM oocytes with human round spermatids and obtained one embryo that developed to 8-cell stage (Table 1).

To exclude parthenogenetic development and confirm the contribution of sperm to the embryos obtained by ROSI of induced spermatids, we performed genetic analysis of the 8-cell embryo by examination of the profile of 13 Combined DNA index System (CODIS) STR loci and sex loci. Parentage analysis assumes that the embryo must receive, in the absence of mutation, one allele matching each parent. STR analysis revealed that all embryonic alleles matched, with a single paternal and maternal allele, respectively (Fig. 5b; Supplementary Information, Fig. S6). In this case, for example, the D16S539 loci of fetal skin (the corresponding fetal gonad was subjected to spermatid generation) had alleles 9 and 9, and the maternal granulosa cells had alleles 10 and 12 . The 8-cell embryo was found to have alleles 9 , 10 and 12 , of which allele 9 was exclusively derived from the spermatid, for the alleles 10 and 12, one is from the oocyte, and the other is from the polar body or residual granulosa cells attached to the embryo during culture. The intact euploid genome of the embryo developed from oocyte injected with an induced spermatid was revealed by whole-genome CNV analysis (Fig. 5c). Thus, these results indicated that testicular organogenesis-derived spermatids could fertilize oocytes and support early embryo development in vitro.

\section{DISCUSSION}

According to cell division type, mammalian cells can be divided into mitotic somatic cells and germ cells able to undergo meiosis. Human organogenesis is a complex process involving cell differentiation and spatiotemporally dynamic intercellular interactions. In particular, organogenesis of the gonadal organs involves both mitosis and meiosis of germ cells and interactions between somatic cells and germ cells. Established in vitro threedimensional stem cell models of human organogenesis, including optic cup, ${ }^{29}$ cortical tissues, ${ }^{30}$ and liver buds, ${ }^{31}$ among others, have enormous potential for modeling normal development and disease, as a tool for drug testing, and as framework for therapeutic testing. These existing models are all of mitotic organs, and there have been no internationally recognized reports of in vitro meiotic organogenesis models of human organs, such as human testis and ovary. Here, we report the first successful in vitro modelling of human testicular organogenesis including development of the seminiferous epithelium and robust generation of functional spermatids that conform to the "Gold Standards" of in vitro-derived germ cells. ${ }^{19}$ Our model recapitulates meiosis in a cultured environment and proves the feasibility of in vitro generation of human sperm by meiosis, which would accelerate research on spermatogenesis disorders and approaches to fertility preservation in prepubertal male tumor patients.

The study of human testis organogenesis in vitro has been limited to date, despite a century of efforts to complete spermatogenesis in culture. Ex vivo organ culture $24,32,33$ and coculture of PGCs with testicular somatic cells ${ }^{25}$ have been successfully achieved in mice, with the derivation of functional sperms and the eventual generation of offspring, but these advancements have not been recapitulated in human. ${ }^{24,32}$ Adding to the complexity, the relatively short duration of murine spermatogenesis (34.5 days) is nearly doubled in human (64 days), ${ }^{4}$ and the first and second meiosis stages last about 3 weeks in human. Our culture method of human embryonic gonadal tissues containing PGCs and immature somatic cells robustly transformed these tissues into mature testis with seminiferous epithelium. In males, human germ cells form during the embryonic stage and start the process of spermatogenesis at puberty after a quiescent period of more than 10 years. $^{34}$ In our model, testicular organogenesis bypassed the quiescent stage and underwent spermatogenesis in vitro in about 30 days, which parallels the duration of the first and second meiosis in vivo.

Most regulators of meiosis are evolutionarily conserved among mammals; therefore, we slightly modified the medium used in our previous method of in vitro mouse meiosis to make it suitable for human gonads. ${ }^{24,25}$ In our culture system, we eliminated RA from the culture medium since the start, because addition of RA to the culture caused severe cell apoptosis and morphologic deformation of the tubule (Supplementary Information, Fig. S3). Meiosis initiated in the absence of RA supplementation, confirming that Sertoli cells have been mature and seminiferous tubule function was successfully recapitulated in vitro.

We previously showed that co-culturing of murine germ cells and testicular somatic cells could achieve spermatogenesis in vitro, albeit limited to a single round of meiosis and associated with loss of spermatogonia self-renewal ability. ${ }^{25}$ In our human testicular organogenesis culture system, the medium was supplemented with GDNF, bFGF, and EGF, as these factors could support the maintenance of spermatogonia self-renewal in vitro. In the present model, a BTB formed during in vitro culture, dividing the basal and adluminal compartments via tight junctions among Sertoli cells, providing an environmental niche supporting spermatogonia maintenance in the basal compartment and meiosis in the adluminal compartment. ${ }^{35}$ Indeed, we obtained a consistent proportion of haploid cells by FACS after more than 100 days of culture. In short, Sertoli cells and germ cells establish an interactive microenvironment that precisely maintains continuous and persistent spermatogenesis.

Meiosis enables homologous recombination to produce genetically unique haploid cells. We found several indications of recombination in our model, including expression of SYCP3 and $\mathrm{YH} 2 \mathrm{AX}$, and the results of STR profiling ${ }^{36}$ of FACS purified in vitro-derived haploid cells. We compared somatic fetal tissues to in vitro-derived haploid cells using several polymorphous STR loci on the same chromosome, detecting chromosomal recombination in our spermatids after meiosis. These cytological and genetic findings support that our haploid cells were produced by in vitro meiosis, and not by aberrant mitosis. Additional wholegenome CNV analysis of FACS-purified haploid cells demonstrated that an intact euploid genome was produced in the in vitro-derived spermatids. Ensuring genomic integrity is essential when exploring potential therapies for the treatment of human infertility, to avoid the formation and propagation of mutations and transgenes.

In this study, in vitro-derived haploid germ cells that formed after meiosis underwent spermiogenesis, a complex process involving dramatic morphological changes as the spermatids mature into spermatozoa. Ultrastructural evidence of this 
a
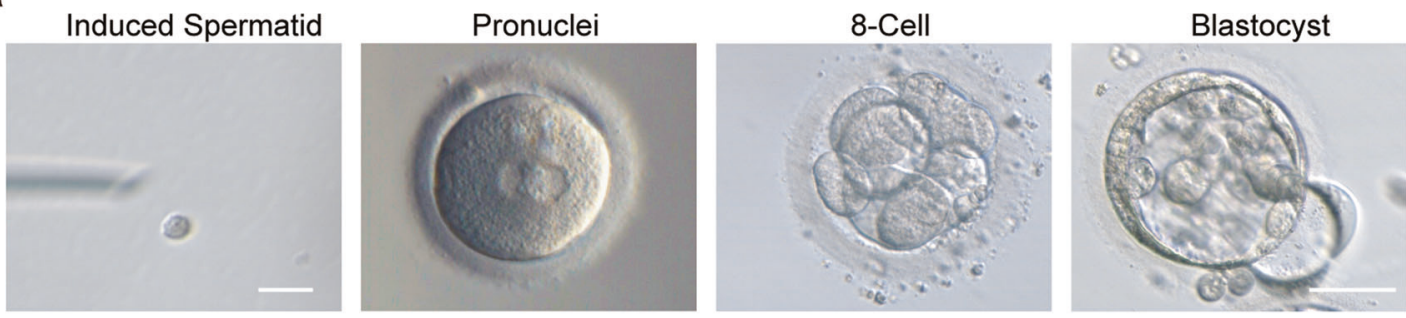

b
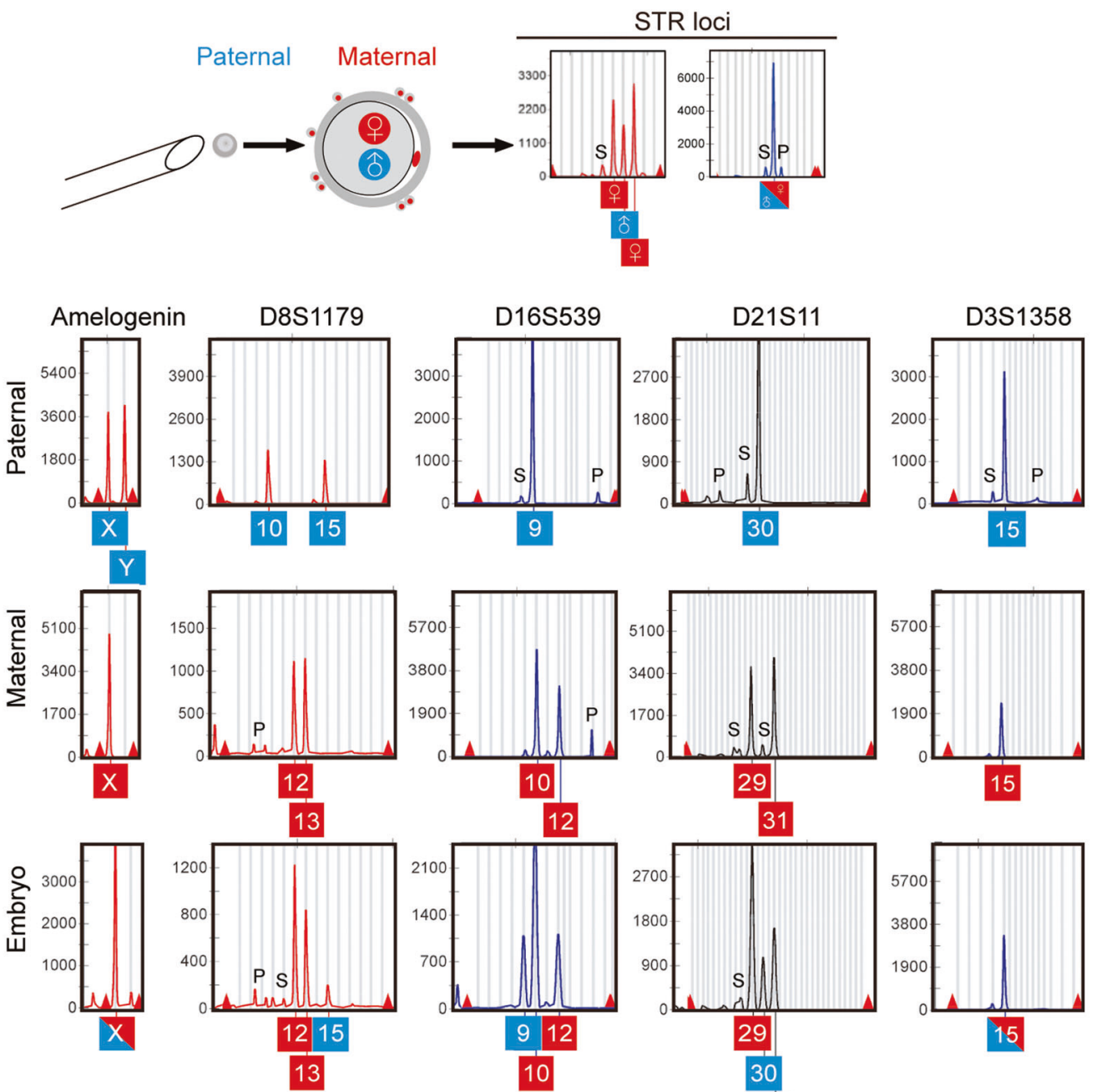

31
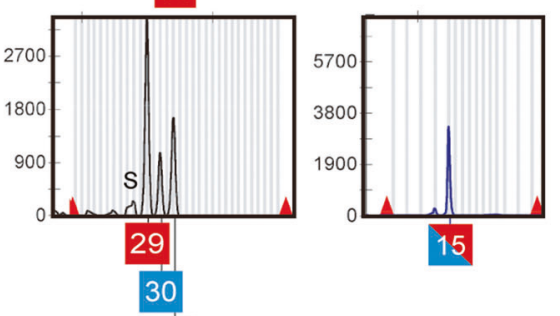

C

Embryo, 44+XX

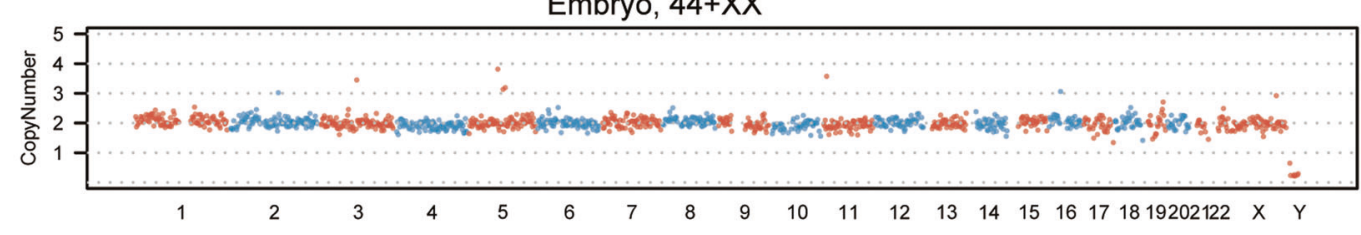

Fig. 5 Functional analysis of spermatids derived from in vitro testicular organogenesis. a A MII oocyte derived through IVM was injected with an induced spermatid, followed by development to pronuclear stage with two pronuclei, 8-cell and blastocyst stages. Scale bars $=10 \mu \mathrm{m}$ (left); $100 \mu \mathrm{m}$ (right). b STR and sex loci of embryo, and fetal skin (paternal) that contributed the round spermatid and granulosa cells (maternal) of oocyte. S: Stutter peak; P: Pull down peak. c CNV analysis of the embryo (b) by genomic DNA sequencing. 
Table 1. Fertilization rate and development of human IVM oocytes with human in vivo round spermatids and in vitro-derived spermatids.

\begin{tabular}{lcll}
\hline Groups & Number of oocytes injected & $\begin{array}{l}\text { Number of fertilized oocytes } \\
\text { with 2PB and 2PN }\end{array}$ & $\begin{array}{l}\text { Number and percentage of } \\
\text { embryos }\end{array}$ \\
\hline Human in vivo round spermatids & 9 & $1(11.11 \%)$ & $1(11.11 \%)$ \\
In vitro-derived spermatids & 16 & $2(12.5 \%)$ & $2(12.5 \%)$ \\
\hline 2PB: 2 polar bodies, 2PN: 2 pronuclei. & & & \\
\hline
\end{tabular}

transformation in our cultured spermatids included early tail structures emanating from the centrioles and acrosome vesicles, whose appearance are two key events of spermiogenesis to form tadpole-shaped sperm.

In our study, ROSI of in vitro-derived spermatids demonstrated that these cells had the capacity to fertilize oocytes followed by pre-implantation development of the resulting embryo to the blastocyst stage. In agreement with the literature, we considered the presence of two pronuclei as evidence of fertilization. ${ }^{37,38}$ We observed similar rates of fertilization and early embryo development between in vitro-derived spermatids (12.5\%) and in vivoderived round spermatid controls $(11.11 \%)$. Fertilization rates following ROSI of IVM oocytes have not been established; however, these rates are within the same range as that $(14 \%)$ calculated from reported ROSI fertilization rate ${ }^{39}$ and IVM oocyte fertilization rate. ${ }^{40}$ This implies that the in vitro-derived spermatids were functionally similar to in vivo spermatids. Polymorphous STR loci analysis to trace the parental sources of genetic DNA of embryos resulting from ROSI confirmed the contribution of in vitro-derived spermatids to embryonic DNA.

In summary, we report a successful model of in vitro human testicular organogenesis from embryonic genital ridge. The ability of this model to form functional seminiferous tubules that support not only spermatogonia self-renewal, but also the maturation of haploid spermatids represents a remarkable breakthrough for reproductive medicine research. To our knowledge, this is the first report of faithful in vitro recapitulation of meiosis from human gonadal germ cells, which enables the formation of functional gametes capable of fertilizing eggs via ROSI, and also complies with the "gold standards"19 for in vitro meiosis, including erasure of imprints, synapsis, and recombination. This model will support basic research aimed at understanding the complexities of testicular organogenesis, and provide a basis for the development of clinical technology much needed for fertility preservation of prepubertal male tumor patients.

\section{MATERIALS AND METHODS}

Origin of human fetal gonads, oocytes, sperm and round spermatids

Written informed consents were obtained from all donors. The fetal gonads were obtained from aborted fetuses with the approval of the Ethical Committee of Nanjing Maternity and Child Health Care Hospital (Permission Number (2017)68). The human sperm were collected from donors according to World Health Organization 2010 guidelines, and the donated immature oocytes and human round spermatids were obtained from patients undergoing assisted reproductive technology, and fertility preservation, respectively, with approval of the Ethical Committee of the First Affiliated Hospital of Nanjing Medical University (Permission Number 2012-SR-128). The experiments involving human samples were also approved by the Ethical Committee of Nanjing Medical University (Permission Number (2017)582). For the fetal gonad, samples with the state of dissolution or congestion, or with genetic defects or infectious diseases, such as Hepatitis B, AIDS and syphilis, were excluded.
Tissue culture

The gonads of the aborted 12- to 19-week fetuses were gently separated into fragments approximately $3 \mathrm{~mm}$ in diameter (5-20 fragments in total) using fine tweezers. A part of these samples were subjected to morphological analysis. Using a standard gasliquid interphase method, gonad tissue fragments were placed on agarose gel stands prepared by pouring heated and dissolved agarose in millipore water $(0.25 \%[\mathrm{w} / \mathrm{v}])$ into a $10 \mathrm{~cm}$ dish. Cooled gels were cut into $8 \times 8 \times 8 \mathrm{~mm}^{3}$ pieces. Gels were soaked in the culture medium for more than $24 \mathrm{~h}$ to replace the water with the medium. One cube of agarose gel was placed in each well of a four-well plate and loaded with one tissue fragment. The culture medium aMEM was supplemented with 10\% KSR, BMP 4/7 (20 ng/ $\mathrm{mL}$, R\&D Systems), SCF (20 ng/mL, R\&D Systems), bFGF $(20 \mathrm{ng} / \mathrm{mL}$, R\&D Systems), EGF ( $20 \mathrm{ng} / \mathrm{mL}, R \& D$ Systems), GDNF $(20 \mathrm{ng} / \mathrm{mL}$, R\&D Systems), Activin A (100 ng/mL, R\&D Systems), testosterone (10 mM, Acros Organics), FSH (200 ng/mL, Sigma), and BPE (50 mg/ $\mathrm{mL}$, Corning Life Sciences), modified from a medium previously described, ${ }^{25}$ with or without RA $\left(10^{-6} \mathrm{M}\right)$. The medium in each well was filled to a level just below the upper surface of the agarose gel stand. Medium was changed every 2 days. Tissues were cultured in $5 \% \mathrm{CO}_{2}$ at $34^{\circ} \mathrm{C}$.

\section{Immunostaining}

Tissues were fixed in 4\% paraformaldehyde (PFA) in PBS overnight at room temperature and embedded in paraffin. Standard immunostaining techniques were used to evaluate $5 \mu \mathrm{m}$ serial sections. Briefly, sections were blocked for $60 \mathrm{~min}$ using $0.3 \%$ Triton-X $100 / 2 \%$ BSA in PBS before incubation overnight at $4{ }^{\circ} \mathrm{C}$ with primary antibodies and 60 min incubation with FITC-, Cy3and Cy5-conjugated secondary antibodies (Jackson Immuno Research).

Co-immunostaining with DDX4 and ZO1 rabbit antibodies was performed by TSA-based IF. ${ }^{41}$ Briefly, sections were incubated at room temperature with the DDX4 rabbit antibody for $2 \mathrm{~h}$, and then incubated with HRP-labeled secondary antibody. Following 3 washes in PBS for $5 \mathrm{~min}$, sections were incubated with TSA-488. Following incubation with the ZO1 rabbit antibody for $2 \mathrm{~h}$, sections were incubated with Cy3-conjugated secondary antibody.

For consecutive immunostaining of the same sections with four rabbit antibodies (DDX4, PLZF, SYCP3 and PRM1), sections were washed with $2 \times$ SSC (sodium chloride $0.6 \mathrm{M}$, sodium citrate 0.06 M) high salt buffer to wash off glycerol, followed by microwave and high temperature treatment for $10 \mathrm{~min}$ to remove the previous antibodies for the next antibody staining. Primary antibodies used in this study are shown in Supplementary Information, Table S4. The DNA was labeled using $10 \mu \mathrm{g} / \mathrm{mL}$ Hoechst 33342 dye for $15 \mathrm{~min}$. Images were captured using a camera-equipped Zeiss LSM800 confocal microscope.

\section{Electron microscopy}

Gonadal and cultured tissue fragments were fixed in $2.5 \%$ gluteraldehyde, $3 \%$ paraformaldehyde with $5 \%$ sucrose in $0.1 \mathrm{M}$ sodium cacodylate buffer ( $\mathrm{pH} 7.4$ ), and post-fixed in $1 \%$ osmium tetroxidein veronal-acetate buffer. Samples were incubated overnight in $0.5 \%$ uranyl acetate in veronal-acetate buffer $(\mathrm{pH} \mathrm{6.0)}$, 
then dehydrated and embedded in Embed-812 resin. Fiftynanometer sections were prepared using a Leica Ultracut UCT microtome with a Diatome diamond knife and stained with uranyl acetate and lead citrate. The sections were examined using a FEI Tecnai Spirit at $80 \mathrm{keV}$.

\section{Flow cytometry analysis}

Two to three tissue fragments were harvested and digested by two-step enzyme digestion method as previously described. ${ }^{42}$ Briefly, tissues were dispersed with $1 \mathrm{mg} / \mathrm{mL}$ collagenase type IV at $37^{\circ} \mathrm{C}$ for $10 \mathrm{~min}$ and followed by $0.25 \%$ trypsin/ $1 \mathrm{mM}$ EDTA digestion at $37^{\circ} \mathrm{C}$ for $10 \mathrm{~min}$. Single cells were re-suspended in PBS supplemented with $1 \%$ BSA, then filtered through a $40-\mu \mathrm{m}$ nylon mesh. For ploidy analysis, single cells were stained with 10 $\mu \mathrm{g} / \mathrm{mL}$ Hoechst 33342 for 20 min and washed thrice with PBS. FACS analysis was performed using the FACS Calibur system (Becton, Dickinson).

\section{$\mathrm{FISH}$}

Samples obtained for FISH were spread on a slide, dehydrated in graded ethanol $(70 \%, 85 \%$, and $100 \%)$ and stored at $-20^{\circ} \mathrm{C}$ before processing. Before hybridization, slides were fixed in Carnoy's fixative (methanol-glacial acetic acid 3:1), decondensed for $3 \mathrm{~min}$ in $1 \mathrm{M} \mathrm{NaOH}$, and fixed once in Carnoy's fixative for $10 \mathrm{~min}$. Slides were dehydrated in graded ethanol (70\%, 85\%, 100\%). Hybridization was performed using the FastFish kit, according to the manufacturer's instructions, with probe set 1 for chromosomes 13 and 21 and probe set 2 for chromosomes $X, Y$ and 18 . Slides were evaluated using a Zeiss710 microscope.

Bisulfite sequencing

Bisulfite sequencing of genomic DNA from human round spermatid and induced spermatids was performed using the EZ DNA Methylation-direct kit (Zymo Research). PCR amplification was performed using TAKARA HS DNA polymerase (TAKARA Ltd; DA LIAN, CHN) with specific primers for the H19 and SNRPN DMR imprinting regions. Primers used in this study are listed in Supplementary Information, Table S3. To determine the methylation state of individual $\mathrm{CpG}$ sites, PCR products were gel extracted, subcloned into the pMD18T vector (TAKARA Ltd; DA LIAN, CHN), and then sequenced. Visualization and quantification of the bisulfite sequence data were analyzed by the web-based tool, QUMA (http://quma.cdb.riken.jp/).

\section{CNV analysis}

A single somatic cell or round spermatid from biopsied specimen or cultured tissue was placed into the lysis buffer by mouth pipette. DNA was extracted using the Single Cell DNA Quick-Amp Kit (Yikon Genomics). Embryonic DNA was extracted using QIAseq FX Single Cell DNA Library Kit (QIAGEN). DNA quality and integrity were assessed by the Qubit2.0 and a Bioanalyzer 2100 (Agilen, Germany). Approximately $300 \mathrm{ng}$ DNA was sheared to approximately 300 bp by Covaris S2, DNA was end repaired, A-tailed and ligated to adapters utilizing the NEBNext Ultra DNA Library Prep Kit for Illumina. The libraries were sequenced on Illumina Hiseq $X$ with $2 \times 150 \mathrm{bp}$ paired-end sequencing, using Hiseq Control Software (HCS). The CNV regions were identified according to the published procedure. ${ }^{43}$

\section{Oocytes collection and in vitro maturation}

Multiple follicular growth was induced using a gonadotropinreleasing hormone $(\mathrm{GnRH})$ agonist and $\mathrm{GnRH}$ antagonist. Human chorionic gonadotropin (hCG) was administered when the dominant follicle reached a maximum diameter of $18 \mathrm{~mm}$. Oocytes were collected under vaginal ultrasound-guided follicle puncture performed $34-36 \mathrm{~h}$ after hCG injection. Immature oocytes, including $\mathrm{Ml}$ or Germinal vesicle (GV) oocytes, were subjected to IVM by culturing in $1 \mathrm{~mL}$ of IVM Medium (SAGE, USA) supplemented with $0.075 \mathrm{IU} / \mathrm{mL} \mathrm{FSH}$ and $0.075 \mathrm{IU} / \mathrm{mL}$ LH overlaid with mineral oil. Oocytes were incubated at $37^{\circ} \mathrm{C}$ in $6 \% \mathrm{CO}_{2}$ for $24 \mathrm{~h}$ and then assessed for nuclear maturity. Oocytes with a polar body were considered as mature (metaphase II).

ROSI, artificial oocyte activation and embryo culture

IVM oocytes were used for ROSI injection. Each ROSI dish was mounted with approximately eight micro droplets $(5 \mu \mathrm{L}$ HTFHEPES medium (SAGE, USA) supplemented with $10 \%$ human serum albumin (HSA)) each loaded with a single mature oocyte. One droplet $(10 \mu \mathrm{L})$ of PVP-based medium (SAGE, USA) was placed adjacent to oocyte droplets and the dish was overlaid with mineral oil. Injections were performed using an Olympus IX71 inverted microscope (Tokyo, Japan) at $\times 400$, equipped with a heated stage (MATS-USMZSS, Thokai Hit, Japan) maintained at $37^{\circ}$ C, using two micromanipulators (Narishige, Tokyo, Japan). Briefly, PVP-equilibrated injection needles (ID $10 \mu \mathrm{m}$ ) were used to collect haploid cells. Oocytes were positioned with the polar body at the 12 o'clock position and a holding pipette (SAGE, USA) at the 9 o'clock position. After zona penetration at the 3 o'clock position, the ooplasm was aspirated with the injection needle and a haploid cell was deposited into the oocyte.

Oocytes were then incubated in fertilization medium (SAGE, USA) at $37^{\circ} \mathrm{C}$ in $6 \% \mathrm{CO}_{2}$ for $30 \mathrm{~min}$, before being processed for artificial oocyte activation. Oocytes were incubated in $1000 \mathrm{~mL}$ of HTF-HEPES medium supplemented with 10\% HSA containing $10 \mathrm{mM}$ calcium ionophore (A23187, Sigma, St. Louis, Mo, USA) at $37^{\circ} \mathrm{C}$ for $15 \mathrm{~min}$, washed three times in HTF-HEPES medium, and then cultured in cleavage medium (SAGE, USA) at $37^{\circ} \mathrm{C}$ in $6 \% \mathrm{CO}_{2}$ humidified gas atmosphere. Pronuclear formation was scored to determine activation after $18 \mathrm{~h}$.

STR analysis

DNA extracted from single round spermatid cells was amplified using multiple displacement amplification. The EX22 kit and $21+$ 1 kit were used to detect STR loci. The EX22 kit simultaneously amplified and detected 22 loci, including 13 CODIS loci (CSF1PO, FGA, TH01, TPOX, vWA, D3S1358, D5S818, D7S820, D8S1179, D13S317, D16S539, D18S51, D21S11) and D2S1338, D6S1043, D12S391, D19S433, D2S441, D10S1248, PentaD, PentaE, and sex loci Amelogenin. The $21+1$ kit detected loci of Amelogenin, D6S474, D22S1045, D12ATA63, D10S1248, D1S1677, D11S4463, D2S441, D1S1627, D3S4529, D6S1017, D4S2408, D19S433, D17S1301, D1GATA113, D18S853, D20S482, D14S1434, D9S1122, D2S1776, D10S1435, and D5S2500. The results were analyzed using GeneMapper ID v3.1.

\section{ACKNOWLEDGEMENTS}

We thank Peng Xiang for the 33-HSD, CYP17A1 antibodies, Fei Gao for the AMH antibody, and Sigrid Eckardt for help with paper preparation. This work was supported by National Key R\&D Program (2017YFA0103803), National Natural Science Foundation of China (31890784), and National Key R\&D Program (2016YFA0500903, 2016YFA0503300).

\section{AUTHOR CONTRIBUTIONS}

Conceptualization, X.G., F.D., Q.Z., and J.S.; Methodology, Q.C., Q.Zeng, Y.W., J.T., J.Y.; Investigation, Y.Y., L.L., H.Z., M.H., J.C., Q.Zhou, R.H., X.W., Z.Z., X.Y., J.H., D.H., J.L.; Writing (original draft), X.G., F.D., Q.Z. and J.S.; Writing (review and editing), X.G., F.D., Q.Z., and J.S.; Funding Acquisition, Y.Y., X.G., F.D., Q.Z., and J.S.; Supervision, X.G., F.D., Q.Z., and J.S.

\section{ADDITIONAL INFORMATION}

Supplementary information accompanies this paper at https://doi.org/10.1038/ s41422-020-0283-z.

Competing interests: The authors declare no competing interests. 


\section{REFERENCES}

1. Chemes, H. E. Infancy is not a quiescent period of testicular development. Int. J. Androl. 24, 2-7 (2001).

2. Rey, R. A., Musse, M., Venara, M. \& Chemes, H. E. Ontogeny of the androgen receptor expression in the fetal and postnatal testis: its relevance on Sertoli cell maturation and the onset of adult spermatogenesis. Microsc. Res. Tech. 72, 787-795 (2009).

3. Stukenborg, J. B., Colon, E. \& Soder, O. Ontogenesis of testis development and function in humans. Sex. Dev. 4, 199-212 (2010).

4. Clermont, Y. Kinetics of spermatogenesis in mammals: seminiferous epithelium cycle and spermatogonial renewal. Physiol. Rev. 52, 198-236 (1972).

5. Inhorn, M. C. \& Patrizio, P. Infertility around the globe: new thinking on gender, reproductive technologies and global movements in the 21st century. Hum. Reprod. Update 21, 411-426 (2015).

6. Stiller, C. A. \& Parkin, D. M. Geographic and ethnic variations in the incidence of childhood cancer. Br. Med. Bull. 52, 682-703 (1996).

7. Hargreave, M. et al. Association between fertility treatment and cancer risk in children. JAMA 322, 2203-2210 (2019).

8. Thomson, A. B. et al. Semen quality and spermatozoal DNA integrity in survivors of childhood cancer: a case-control study. Lancet 360, 361-367 (2002).

9. Ginsberg, J. P. Educational paper: the effect of cancer therapy on fertility, the assessment of fertility and fertility preservation options for pediatric patients. Eur. J. Pediatrics 170, 703-708 (2011).

10. Martinez, G. et al. Impact of Hodgkin or non-Hodgkin lymphoma and their treatments on sperm aneuploidy: a prospective study by the French CECOS network. Fertil. Steril. 107, 341-350 e345 (2017).

11. Chatterjee, R., Mills, W., Katz, M., McGarrigle, H. H. \& Goldstone, A. H. Germ cell failure and Leydig cell insufficiency in post-pubertal males after autologous bone marrow transplantation with BEAM for lymphoma. Bone Marrow Transplant. 13, 519-522 (1994).

12. Ji, D. et al. Somatic mutations and immune alternation in rectal cancer following neoadjuvant chemoradiotherapy. Cancer Immunol. Res. 6, 1401-1416 (2018).

13. Rakici, S. Y., Bedir, R. \& Hatipoglu, C. Are there predictors that can determine neoadjuvant treatment responses in rectal cancer? Turkish J. Gastroenterol. https://doi.org/10.5152/tjg.2018.18179 (2018).

14. Lee, I. H. et al. Genetic variations using whole-exome sequencing might predict response for neoadjuvant chemoradiotherapy in locally advanced rectal cancer. Med. Oncol. 35, 145 (2018).

15. de Lambert, G., Poirot, C., Guerin, F., Brugieres, L. \& Martelli, H. Preservation of fertility in children with cancer. Bull. du cancer 102, 436-442 (2015).

16. Levine, J. Fertility preservation in children and adolescents with cancer. Minerva Pediatrica 63, 49-59 (2011).

17. Mitchell, R. T., Saunders, P. T., Sharpe, R. M., Kelnar, C. J. \& Wallace, W. H. Male fertility and strategies for fertility preservation following childhood cancer treatment. Endocr. Dev. 15, 101-134 (2009).

18. Tournaye, H., Dohle, G. R. \& Barratt, C. L. Fertility preservation in men with cancer. Lancet 384, 1295-1301 (2014).

19. Handel, M. A., Eppig, J. J. \& Schimenti, J. C. Applying "gold standards" to in-vitroderived germ cells. Cell 157, 1257-1261 (2014).

20. Tang, W. W. et al. A unique gene regulatory network resets the human germline epigenome for development. Cell 161, 1453-1467 (2015)

21. Li, L. et al. Single-cell RNA-Seq analysis mmaps development of human germline cells and gonadal niche interactions. Cell Stem Cell 20, 858-873 e854 (2017).
22. Costoya, J. A. et al. Essential role of Plzf in maintenance of spermatogonial stem cells. Nat. Genet. 36, 653-659 (2004).

23. Sasaki, K. et al. The germ cell fate of Cynomolgus monkeys is specified in the Nascent Amnion. Dev. Cell 39, 169-185 (2016).

24. Sato, T. et al. In vitro production of functional sperm in cultured neonatal mouse testes. Nature 471, 504-507 (2011).

25. Zhou, Q. et al. Complete meiosis from embryonic stem cell-derived germ cells in vitro. Cell Stem Cell 18, 330-340 (2016).

26. Nistal, M., Abaurrea, M. A. \& Paniagua, R. Morphological and histometric study on the human Sertoli cell from birth to the onset of puberty. J. Anat. 134, 351-363 (1982).

27. Habert, R., Lejeune, H. \& Saez, J. M. Origin, differentiation and regulation of fetal and adult Leydig cells. Mol. Cell. Endocrinol. 179, 47-74 (2001).

28. Syrjanen, J. L., Pellegrini, L. \& Davies, O. R. A molecular model for the role of SYCP3 in meiotic chromosome organisation. elife 3, https://doi.org/10.7554/ eLife.02963 (2014).

29. Nakano, T. et al. Self-formation of optic cups and storable stratified neural retina from human ESCs. Cell Stem Cell 10, 771-785 (2012).

30. Lancaster, M. A. et al. Cerebral organoids model human brain development and microcephaly. Nature 501, 373-379 (2013).

31. Takebe, T. et al. Vascularized and functional human liver from an iPSC-derived organ bud transplant. Nature 499, 481-484 (2013).

32. Gohbara, A. et al. In vitro murine spermatogenesis in an organ culture system. Biol. Reprod. 83, 261-267 (2010).

33. Reda, A. et al. In vitro differentiation of rat spermatogonia into round spermatids in tissue culture. Mol. Hum. Reprod. 22, 601-612 (2016).

34. Rey, R. A. Mini-puberty and true puberty: differences in testicular function. Annales d'endocrinologie 75, 58-63 (2014).

35. Iliadou, P. K., Tsametis, C., Kaprara, A., Papadimas, I. \& Goulis, D. G. The sertoli cell: novel clinical potentiality. Hormones 14, 504-514 (2015).

36. Tracey, M. Short tandem repeat-based identification of individuals and parents. Croatian Med. J. 42, 233-238 (2001).

37. van der Westerlaken, L., Helmerhorst, F., Dieben, S. \& Naaktgeboren, N. Intracytoplasmic sperm injection as a treatment for unexplained total fertilization failure or low fertilization after conventional in vitro fertilization. Fertil. Steril. 83, 612-617 (2005).

38. Van Landuyt, L. et al. Blastocyst formation in in vitro fertilization versus intracytoplasmic sperm injection cycles: influence of the fertilization procedure. Fertil. Steril. 83, 1397-1403 (2005).

39. Vloeberghs, V., Verheyen, G. \& Tournaye, H. Intracytoplasmic spermatid injection and in vitro maturation: fact or fiction. Clinics 68, 151-156 (2013).

40. Pongsuthirak, P., Songveeratham, S. \& Vutyavanich, T. Comparison of blastocyst and Sage media for in vitro maturation of human immature oocytes. Reprod. Sci. 22, 343-346 (2015).

41. Kosmac, K. et al. Immunohistochemical identification of human skeletal muscle macrophages. Bio-protocol 8, https://doi.org/10.21769/BioProtoc.2883 (2018).

42. O'Brien, D. A. Isolation, separation, and short-term culture of spermatogenic cells. Male Reproductive Toxicol. 3A, 246-264 (1993).

43. Zong, C., Lu, S., Chapman, A. R. \& Xie, X. S. Genome-wide detection of singlenucleotide and copy-number variations of a single human cell. Science 338, 1622-1626 (2012). 